\title{
A Note on the Pressure Equations Used in Zone Fire Modeling
}

Ronald G. Rehm Glenn P. Forney

$-Q C$ 100 .456 4906 1992 C. 2 



\section{A Note on the Pressure Equations Used in Zone Fire Modeling}

Ronald G. Rehm

Computing and Applied Mathematics Laboratory

Glenn P. Forney

Building and Fire Research Laboratory

August 1992

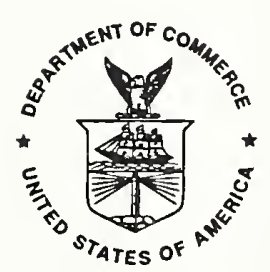

\section{U.S. Department of Commerce}

Barbara Hackman Franklin, Secretary

Technology Administration

Robert M. White, Under Secretary for Technology

National Institute of Standards and Technology

John W. Lyons, Director 



\section{Contents}

1 Introduction 1

2 Conservation Equations

3 Pressure Equation One-Room Model 5

3.1 Phase Plane Analysis for One-Room Model . . . . . . . . . . 7

4 Pressure Equation Two-Room Model 9

4.1 Phase Plane for Two-Room Model . . . . . . . . . . . . 11

4.2 Zero-Order Singular-Perturbation Analysis . . . . . . . . . 14

$\begin{array}{lll}5 & \text { Conclusions } & 17\end{array}$

$\begin{array}{ll}\text { A Zone-Fire Model Stiffness } & 19\end{array}$

A.1 A Derivation Illustrating the Presense of Stiffness . . . . . . . . . . 19

A.2 Some Numerical Considerations . . . . . . . . . . . . . . . . . . 24

B Mathematica Commands Used to Perform Calculations and to Produce Figures

\section{List of Figures}

1 One Room Test Case Configuration . . . . . . . . . . . . . 6

2 Solution of a Non-Dimensionalized Pressure Differential Equation for Initial Conditions: $p(0)=-2, p(0)=0$ and $p(0)=2 \ldots . . . .7$

3 Two Room Test Case Configuration . . . . . . . . . . . 10

4 Solution Plot of Equation 12 for $a=1, b=2$ and Initial Conditions $x(0)=y(0)=0 \ldots \ldots \ldots \ldots$

5 Solution Plot of Equation 12 for $a=4, b=2$ and Initial Conditions $x(0)=y(0)=0 \ldots \ldots \ldots \ldots$

6 Solution Plot of Equation 12 for $a=0.1, b=2$ and Initial Conditions

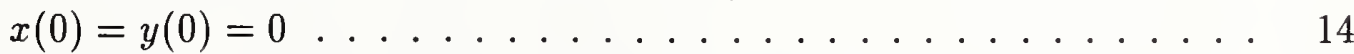

7 Phase Plane Plot of Equation 12 for $a=1, b=2 \ldots \ldots$

8 Phase Plane Plot of Equation 12 for $a=4, b=2 \ldots \ldots$

9 Phase Plane Plot of Equation 12 for $a=0.1, b=2 \ldots \ldots$ 


\section{List of Tables}

1 Conservative Zone Modeling Differential Equations ......... 5 


\title{
A Note on the Pressure Equations Used in Zone Fire Modeling
}

\author{
Ronald G. Rehm \\ Computing and Applied Mathematics Laboratory \\ Glenn P. Forney \\ Building and Fire Research Laboratory \\ National Institute of Standards and Technology \\ Gaithersburg, MD 20899
}

\begin{abstract}
Examples of simple zone fire models are analyzed. These models illustrate the nature of the numerical problems commonly encountered in zone models of enclosure fires. Often these difficulties arise in the solution of the equations for the pressure in connected rooms because the pressure equilibrates much more rapidly than other dynamical variables. Since these models are very simple, analytical techniques can be applied and some insight gained regarding the nature of these problems. The models consist of ordinary differential equations coupled with algebraic equations. Singular perturbation methods and phase plane analyses, together with numerical integration of the appropriately nondimensionalized equations, are employed to examine the stiff nature of the equations associated with these models. We conclude that many of the difficulties associated with numerical integration of zone fire models in general may be circumvented by appropriate analysis of the zone fire model equations.
\end{abstract}

\section{Introduction}

There is a long history of analysis of the dynamical behavior of fires in buildings using mathematical models. The reason for development of the mathematical models and their use in practice has been reviewed in [1]. The original mathematical model of a plume used in zone fire models was developed by Morton, Taylor and Turner [2]. Other early work contributing to the basic development of these models includes experimental [3] and theoretical [4] studies of the effects of flow through openings induced by fires in enclosures; analytical examples of the development of a stratified 
ceiling layer and the filling of an enclosure by the heated gases [5]; analytical examples of two layer modeling of the smoke movement in two-room structures [6]; and theoretical study of the flow of smoke and hot gases through vents [7].

In this brief introduction, we do not attempt a complete review of the literature on zone fire models, but rather try to give a perspective on where the analysis presented contributes to these efforts. Mathematical models of fires have commonly been divided into two categories, field models and zone models. A description of each of these types of model as well as their relative advantages and disadvantages has also been discussed in [1]. A careful derivation of the equations used in zone fire models from the basic conservation laws utilizing a control-volume approach has been given in [8]. More recently, Forney and Moss [9] have discussed some of the difficulties long encountered in trying to integrate numerically the zone fire models for general problems of interest in the fire community. Since zone models have been so useful to fire engineers and researchers, and since numerical integration of the various equations used in these models has persistently been plagued by difficulties, the current research is presented.

Examples of simple zone models are analyzed. The first of these models is for the pressure in a single room heated by a fire and vented to the outside by a small leak. This model has been solved earlier [5] [9] [10] and provides the basis for analyzing more complex models. Here the single ordinary differential equation (ODE) for this model is made dimensionless, and the nondimensionalization is found to be helpful for analysis of more complex models.

The second model, the coupled pressure equations for two rooms connected to each other and to the outside, was derived in the report of Forney and Moss [9] because it illustrates in very simple form the nature of the problem encountered commonly in zone models of enclosure fires. Here, the equations for this two-room model are made dimensionless, and we analyze them both by asymptotic and by phase-plane methods. The methods provide insight into the nature of the numerical problems commonly encountered in zone models.

Two appendices are also included. In the first, we provide a derivation of a set of differential equations which govern all of the dependent variables (room pressure, layer height, layer temperatures and densities, etc.) for a simple two-layer zone-fire model. In contrast to the examples given in the main body of this report, this one demonstrates that the equations already exhibit stiffness when the pressure equation is coupled to the equations governing the other variables; the reason for this stiffness is that the pressure generally equilibrates rapidly compared to the rate at which the other variables, e.g., layer height, change in an enclosure fire. In the second appendix, we present the commands in Mathematica [11] used to perform the calculations and to produce the figures presented in this report. 


\section{Conservation Equations}

The differential equation for the pressure in a simple zone fire model is derived in this section using the laws of conservation of mass and energy together with the equation of state for an ideal gas. Differential equations for other quantities found in a zone fire model such as temperature or density are derived in the general case in [8] [9]; a set of differential equations for a specific, simple two-layer model in a single enclosure are derived in Appendix A. A zone may consist of a number of interior regions (usually an upper or a lower gas layer). The basic assumption of a zone fire model is that properties such as temperatures can be approximated as uniform throughout the zone. It is remarkable that this assumption seems to hold for as few as two gas layers, which is the model considered in this paper.

Many differential equation formulations based upon these assumptions can be derived. One formulation can be converted into another using definitions of density, internal energy and the ideal gas law. One property that many of these formulations share is the presence of multiple time scales. Physically, the pressure in a compartment equilibrates much quicker than densities and temperatures, see Appendix A. Numerically, this property is known as stiffness and in general requires the use of special differential equation solvers to generate efficient solutions. The main focus of this paper then is to show how an appropriate nondimensionalization for the pressure equations in a zone fire model can be used with analytic techniques such as phaseplane analysis and singular perturbation methods to expose and exploit the presence of these multiple time scales.

Each differential formulation can be expressed in terms of mass and enthalpy flow rates denoted $\dot{m}_{U}, \dot{m}_{L}, \dot{q}_{U}$ and $\dot{q}_{L}$ where the subscripts $L$ and $U$ refer to the lower and upper layer respectively. These flow rates represent the net exchange of mass or energy between zones due to physical phenomena or sub-models such as fire plumes, natural and forced vents, convective, radiative heat transfer, etc. For example, a vent exchanges mass and energy between zones in connected rooms, a fire plume typically adds heat to the upper layer and transfers entrained mass and energy from the lower to the upper layer, and convection transfers energy from the gas layers to the surrounding walls.

As illustrated in Figure 1, a compartment can be divided into two control volumes, an upper layer of hot gases and smoke, and a lower layer of air. The fire produces a plume and acts as a pump to transfer mass from the lower to the upper layer, adding energy to the transferred fluid. The two layer model is quite adequate for many applications because upper and lower layers as described are often observed experimentally in room fires. The gas in each layer has attributes of mass, internal energy, density, temperature, and volume denoted respectively by $m_{i}, E_{i}, \rho_{i}, T_{i}$, and $V_{i}$ where $i=L$ for the lower layer and $i=U$ for the upper layer. The compartment as 
a whole has the attribute of pressure $P$. These eleven variables are related by means of the following seven constraints

$$
\begin{aligned}
\rho_{i} & =\frac{m_{i}}{V_{i}} \text { (density) }, i=L, U \\
E_{i} & =c_{v} m_{i} T_{i} \text { (internal energy) ,i=L,U } \\
P & =R \rho_{i} T_{i} \text { (ideal gas law) }, i=L, U \\
V & =V_{L}+V_{U} \text { (total volume) }
\end{aligned}
$$

The specific heats at constant volume and at constant pressure, $c_{v}$ and $c_{p}$, the universal gas constant, $R$, and the ratio of specific heats, $\gamma$, are related by

$$
\begin{aligned}
\gamma & =\frac{c_{p}}{c_{v}}, \\
R & =c_{p}-c_{v} .
\end{aligned}
$$

The first law of thermodynamics states that the rate of increase of layer internal energy plus the rate at which the layer does work by expansion is equal to the rate at which enthalpy is added to the gas (where we consider the enthalpy added as that from any sources minus losses to the walls). In differential equation form this is

$$
\overbrace{\frac{d E_{i}}{d t}}^{\text {internal energy }}+\overbrace{P \frac{d V_{i}}{d t}}^{\text {work }}=\overbrace{\dot{q}_{i}}^{\text {enthalpy }} .
$$

A differential equation for pressure can be derived by adding the upper and lower layer versions of Eq.(5), noting that $\frac{d V_{U}}{d t}=-\frac{d V_{L}}{d t}$ and

$$
\frac{d E_{i}}{d t}=\frac{d\left(c_{v} m_{i} T_{i}\right)}{d t}=\frac{c_{v}}{R} \frac{d}{d t}\left(P V_{i}\right)
$$

to obtain

$$
\frac{d P}{d t}=\frac{\gamma-1}{V}\left(\dot{q}_{L}+\dot{q}_{U}\right)
$$

Differential equations for the layer volumes, energy, density and temperature can be derived from definitions (1) to (4) using the quotient and product rules. These differential equations are derived in [9] and are summarized in Table 1. Notice that a $\frac{d P}{d t}$ term occurs in all but the mass equations. Handling the pressure equation properly is then crucial for solving the zone fire model both correctly and efficiently.

In the following sections, we examine the pressure equations which arise in some simple examples of single and multi-room zone-fire models. The appropriate nondimensionalization of the pressure equations helps to reveal the analytical character of the equations and allows us to use analytical techniques to examine the nature of the stiffness that arises from the pressure equations. 
Table 1: Conservative Zone Modeling Differential Equations

\begin{tabular}{|c|c|}
\hline Equation Type & Differential Equation \\
\hline \hline mass of layer i & $\frac{d m_{i}}{d t}=\dot{m}_{i}$ \\
\hline pressure & $\frac{d P}{d t}=\frac{\gamma-1}{V}\left(\dot{q}_{L}+\dot{q}_{U}\right)$ \\
\hline energy of layer i & $\frac{d E_{i}}{d t}=\frac{1}{\gamma}\left(\dot{q}_{i}+V_{i} \frac{d P}{d t}\right)$ \\
\hline volume of layer i & $\frac{d V_{i}}{d t}=\frac{1}{\gamma P}\left((\gamma-1) \dot{q}_{i}-V_{i} \frac{d P}{d t}\right)$ \\
\hline density of layer i & $\frac{d \rho_{i}}{d t}=\frac{-1}{c_{p} T_{i} V_{i}}\left(\left(\dot{q}_{i}-c_{p} \dot{m}_{i} T_{i}\right)-\frac{V_{i}}{\gamma-1} \frac{d P}{d t}\right)$ \\
\hline temperature of layer i & $\frac{d T_{i}}{d t}=\frac{1}{c_{p} \rho_{i} V_{i}}\left(\left(\dot{q}_{i}-c_{p} \dot{m}_{i} T_{i}\right)+V_{i} \frac{d P}{d t}\right)$ \\
\hline
\end{tabular}

\section{Pressure Equation One-Room Model}

In Section 3.3.1 of [9], the first example is presented to illustrate the nature of the problems encountered with the integration of the pressure in zone fire modeling. This example is illustrated in Figure 1 (Figure 3 of [9]) and is for a fire in a single room with a small leak near the floor. The major approximations made in this model are that the heated gases never exit through the vent (i.e. the vent is near the floor, while the heated gas layer stays above the leak) and that there is no heat transfer to the walls; i.e. the enclosure has adiabatic boundaries. Under these conditions, the mass loss through the vent equals a constant times the square root of the pressure difference across the vent, and the enthalpy loss through the vent is a constant times the mass loss. The starting equation for this model is Eq. (13) of [9] which is equivalent to Eq. (7), derived earlier. We repeat the analysis given there in nondimensional form and with a slightly different notation because the nondimensionalization gives some additional insight and because this example, which can be solved analytically, forms the basis for analysis of more complicated cases.

$$
\frac{d \tilde{p}}{d \tilde{t}}=\frac{\gamma-1}{V}\left(\dot{q}_{\text {fire }}-\dot{q}_{\text {vent }}\right)
$$

Here the notation is as defined in [9] except that $\tilde{p}$ is the pressure in the room relative to the ambient pressure outside the room $\tilde{p}=\tilde{p}_{e n c}-\tilde{p}_{a t m}$, and $\tilde{t}$ is the time. $\dot{q}_{\text {fire }}$ is the constant fire (heat) source, and $\dot{q}_{v e n t}$ is the enthalpy loss through the vent, defined 


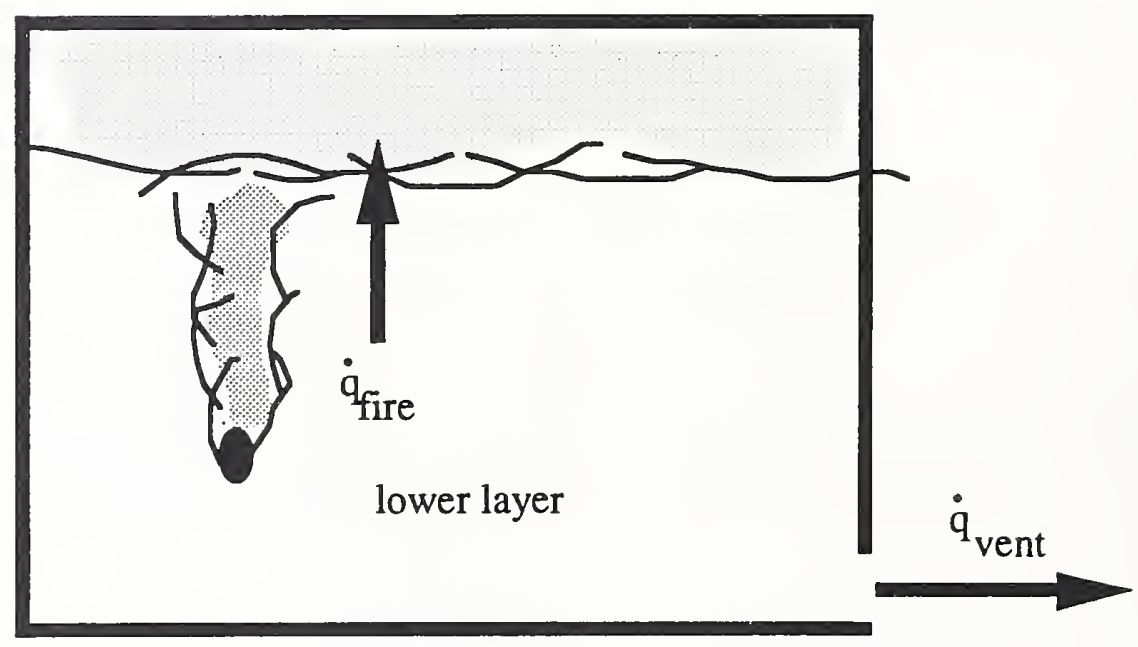

Figure 1: One Room Test Case Configuration

as

$$
\dot{q}_{v e n t}=C_{p} T_{\text {vent }} c_{v e n t} A_{\text {vent }} \operatorname{sgn}(\tilde{p}) \sqrt{2 \rho_{\text {vent }}|\tilde{p}|}
$$

The initial conditions are that $\tilde{p}=0$ at $\tilde{t}=0$.

Now use the following quantities with which to nondimensionalize:

$$
\begin{aligned}
\tilde{p}_{\infty} & =\left(\frac{\dot{q}_{f i r e}}{C_{p} T_{\text {vent }} c_{\text {vent }} A_{\text {vent }} \sqrt{2 \rho_{\text {vent }}}}\right)^{2} \\
\tau & =\frac{V \tilde{p}_{\infty}}{(\gamma-1) \dot{q}_{\text {fire }}}=\frac{V}{(\gamma-1)} \frac{\dot{q}_{\text {fire }}}{\left(C_{p} T_{\text {vent }} c_{\text {vent }} A_{\text {vent }} \sqrt{2 \rho_{\text {vent }}}\right)^{2}}
\end{aligned}
$$

The quantity $\tilde{p}_{\infty}=\tilde{p}_{\text {final }}-\tilde{p}_{a t m}$ is the asymptotic pressure rise in the enclosure relative to the ambient pressure due to the specified heating with the enclosure leak specified. Similarly, the quantity $\tau$ is the time scale over which the pressure rise occurs. These are the proper scaling parameters with which to make the dependent and independent variables nondimensional:

$$
\begin{aligned}
p & =\frac{\tilde{p}}{\tilde{p}_{\infty}} \\
t & =\frac{\tilde{t}}{\tau}
\end{aligned}
$$




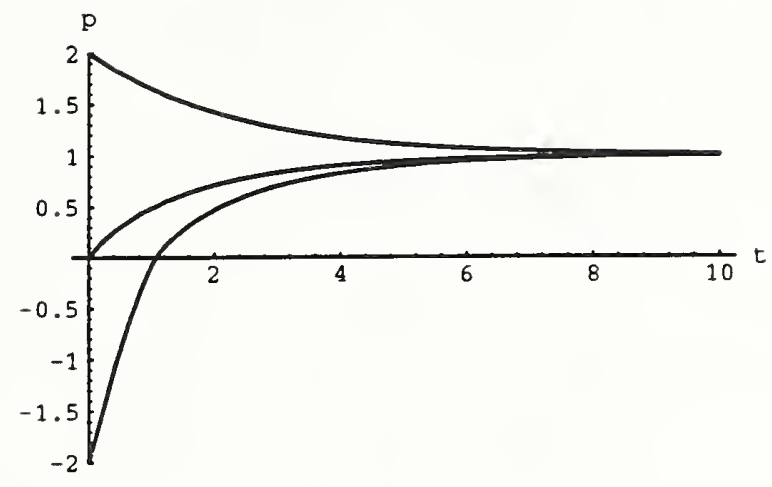

Figure 2: Solution of a Non-Dimensionalized Pressure Differential Equation for Initial Conditions: $p(0)=-2, p(0)=0$ and $p(0)=2$

Then the equation for the pressure becomes

$$
\frac{d p}{d t}=1-\operatorname{sgn}(p) \sqrt{|p|}
$$

The solution to this equation with the initial conditions that $p=0$ at $t=0$ was given earlier in [9] and in [10].

The value of the nondimensionalization will be apparent in the second example, the two-room model, described below.

Figure 2 shows solution plots to Eq. (9) for three values of the initial conditions, $p(0)=2.0,0.0$ and -2.0 . These plots were generated using the software package Mathematica[11], particularly the command NDSolve. The initial condition that $p \equiv y=0.0$ for $t \equiv x=0.0$ is the base calculation performed both in [10] and in [9]. For all initial conditions shown, the solutions all converge at long time to $p(t \rightarrow \infty)=1$, the stable equilibrium solution to the problem.

\subsection{Phase Plane Analysis for One-Room Model}

The phase plane for the one-room model is just the line, but it is instructive to consider it. We will examine first the case of positive dimensionless pressure and study the solution for various initial conditions. Then in the latter portion of this section, we examine also solutions for negative initial dimensionless pressures. In all 
cases, the solutions pass, after long time, to the stable equilibrium value of $p=1$, which is called the fixed point of the equation.

To solve Eq. (9) generally for positive initial pressures, let $p=x^{2}$, and rewrite Eq. (9)

$$
\begin{aligned}
\frac{d p}{1-\sqrt{p}}=d t & =\frac{2 x d x}{1-x} \\
& =2\left(\frac{1}{1-x}-1\right) d x
\end{aligned}
$$

Integrating,

$$
t-t_{0}=-2 \ln |1-x|-2 x+C
$$

where $C$ is the integration constant, and where $x<0$ as well as $x>0$.

Now, let the initial conditions be $\tilde{p}_{\text {enc }}\left(\tilde{t}_{0}\right)=\tilde{p}_{\text {enc } 0}$ at time $\tilde{t}=\tilde{t}_{0} \equiv 0$, where we retain the symbol $\tilde{t}_{0}$ for use later. Then $\tilde{p}_{0}=\tilde{p}_{e n c}-\tilde{p}_{a t m}$ and $p_{0} \equiv \tilde{p}_{0} / \tilde{p}_{\infty}=$ $\left(\tilde{p}_{e n c}-\tilde{p}_{a t m}\right) / \tilde{p}_{\infty}$. Now, since $\tilde{p}_{0}<0$ as well as $\tilde{p}_{0}>0$, and since $\tilde{p}_{\infty}$ can be arbitrarily small, $-\infty<p_{0}<\infty$. Hence the phase space for the one-room model is the whole line.

For $0<p_{0}<\infty$,

$$
t-t_{0}=2 \ln \frac{\left|1-\sqrt{p_{0}}\right|}{|1-\sqrt{p}|}+2\left(\sqrt{p_{0}}-\sqrt{p}\right)
$$

and the phase diagram for either $p_{0}>1$ or for $p_{0}<1$ is the directed line segment from $p_{0}$ to unity.

The solution to Eq. (9) for negative initial dimensionless pressures can be found as follows. For $-\infty<p_{0}<0$,

$$
\frac{d p}{d t}=1+\sqrt{|p|}
$$

Using $p=-x^{2}$,

$$
\frac{d p}{1+\sqrt{|p|}}=d t=2\left(\frac{1}{1+x}-1\right) d x
$$

or, integrating,

$$
t-t_{0}=2 \ln |1+x|-2 x+C^{\prime}
$$

where $C^{\prime}$ is the integration constant, and where $x>0$. 
Now, initially, at $t=0, p=p_{0}$. Hence,

$$
t=2 \ln \frac{1+\sqrt{|p|}}{1+\sqrt{\left|p_{0}\right|}}+2\left(\sqrt{\left|p_{0}\right|}-\sqrt{|p|}\right)
$$

When $p$ starts at negative values, it rises to zero at $t=t_{c}$,

$$
t_{c}=2 \ln \frac{1}{1+\sqrt{\left|p_{0}\right|}}+2 \sqrt{\left|p_{0}\right|}
$$

For $t>t_{c}, p>0$, and we use the solution for $p>0$ with initial conditions that $p=0$ at $t=t_{c}$, namely,

$$
t-t_{c}=2 \ln \frac{1}{|1-\sqrt{p}|}-2 \sqrt{p}
$$

In this case, the phase diagram is a directed line segment from $p_{0}<0$ to unity.

\section{Pressure Equation Two-Room Model}

The second example, and the more interesting one since it illustrates the structure of the mathematical problem in solving the pressure equations, is illustrated in Figure 3 (Figure 6 of [9]). In this example, there are two rooms. In the first room, denoted by subscript 1 , there is a fire and two vents, one to the outside, which is denoted vent 1 , and the second vent, denoted vent 2 , to the second room. Again, the walls are assumed to be adiabatic. The equations for this example are:

$$
\begin{aligned}
& \frac{d \tilde{p}_{1}}{d \tilde{t}}=\frac{\gamma-1}{V_{1}}\left(\dot{q}_{f i r e}-\dot{q}_{\text {vent } 1}-\dot{q}_{\text {vent } 2}\right) \\
& \frac{d \tilde{p}_{2}}{d \tilde{t}}=\frac{\gamma-1}{V_{2}}\left(\dot{q}_{\text {vent } 2}\right)
\end{aligned}
$$

where

$$
\begin{aligned}
& \dot{q}_{v e n t 1}=C_{p} T_{\text {vent } 1} c_{\text {vent } 1} A_{\text {vent } 1} \operatorname{sgn}\left(\tilde{p}_{1}\right) \sqrt{2 \rho\left|\tilde{p}_{1}\right|} \\
& \dot{q}_{\text {vent } 2}=C_{p} T_{\text {vent } 2} c_{\text {vent } 2} A_{\text {vent } 2} \operatorname{sgn}\left(\tilde{p}_{1}-\tilde{p}_{2}\right) \sqrt{2 \rho\left|\tilde{p}_{1}-\tilde{p}_{2}\right|}
\end{aligned}
$$

We define the following scaling parameters:

$$
\tilde{p}_{\infty 11}=\left(\frac{\dot{q}_{f i r e}}{C_{p} T_{v e n t 1} c_{v e n t 1} A_{v e n t 1} \sqrt{2 \rho}}\right)^{2}
$$




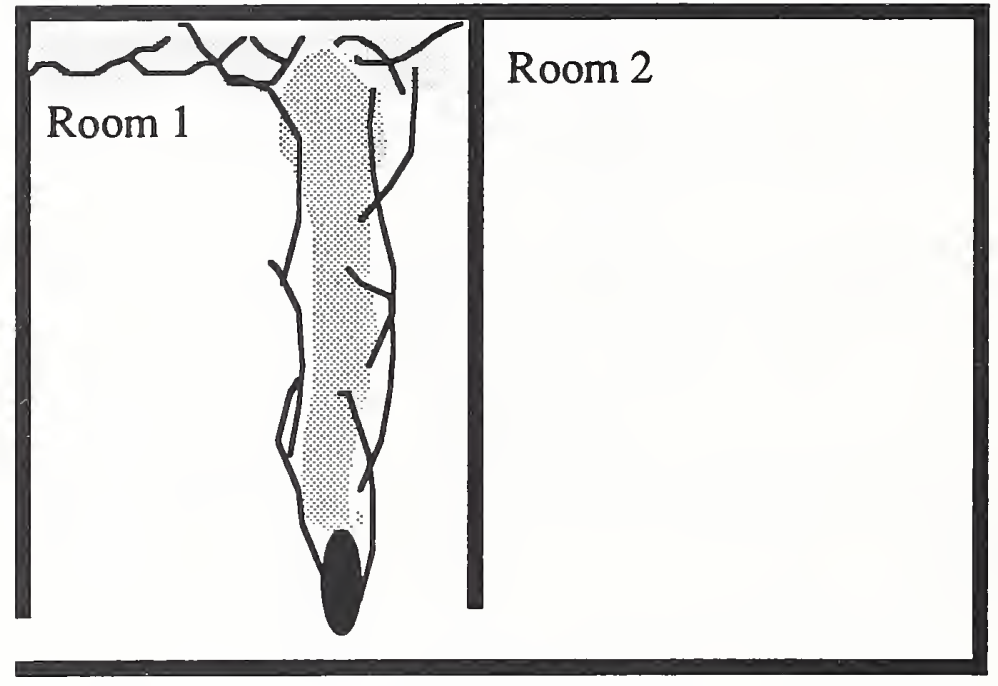

Figure 3: Two Room Test Case Configuration

$$
\begin{aligned}
\tilde{p}_{\infty 22} & =\left(\frac{\dot{q}_{\text {fire }}}{C_{p} T_{\text {vent } 2} c_{\text {vent } 2} A_{\text {vent } 2} \sqrt{2 \rho}}\right)^{2} \\
\tau_{1} & =\frac{V_{1} \tilde{p}_{\infty 1}}{(\gamma-1) \dot{q}_{\text {fire }}}=\frac{V_{1}}{(\gamma-1)} \frac{\dot{q}_{\text {fire }}}{\left(C_{p} T_{\text {vent } 1} C_{\text {vent } 1} A_{\text {vent } 1} \sqrt{2 \rho}\right)^{2}} \\
\tau_{2} & =\frac{V_{2} \tilde{p}_{\infty 2}}{(\gamma-1) \dot{q}_{\text {fire }}}=\frac{V_{2}}{(\gamma-1)} \frac{\dot{q}_{\text {fire }}}{\left(C_{p} T_{\text {vent } \left.2 c_{\text {vent } 2} A_{\text {vent } 2} \sqrt{2 \rho}\right)^{2}}\right.}
\end{aligned}
$$

We use the pressure scale and the time scale associated with vent one as the basic scaling parameters with which to make the equations dimensionless. Define

$$
\begin{aligned}
p_{1} & =\tilde{p}_{1} / \tilde{p}_{\infty 1} \\
p_{2} & =\tilde{p}_{2} / \tilde{p}_{\infty 1} \\
t & =\tilde{t} / \tau_{1}
\end{aligned}
$$

Then, the ratios $\tilde{p}_{\infty 1} / \tilde{p}_{\infty 2}$ and $\tau_{1} / \tau_{2}$ appear in the dimensionless equations, which we can write as follows

$$
\begin{aligned}
& \frac{d p_{1}}{d t}=1-\operatorname{sgn}\left(p_{1}\right) \sqrt{\left|p_{1}\right|}-\sqrt{\frac{\tau_{1} V_{2}}{\tau_{2} V_{1}}} \operatorname{sgn}\left(p_{1}-p_{2}\right) \sqrt{\left|p_{1}-p_{2}\right|} \\
& \frac{d p_{2}}{d t}=\frac{V_{1}}{V_{2}} \sqrt{\frac{\tau_{1} V_{2}}{\tau_{2} V_{1}}} \operatorname{sgn}\left(p_{1}-p_{2}\right) \sqrt{\left|p_{1}-p_{2}\right|}
\end{aligned}
$$




\subsection{Phase Plane for Two-Room Model}

For the phase-plane analysis, change variables to $p_{1}, \Delta p=p_{1}-p_{2}$. Then these equations become

$$
\begin{aligned}
\frac{d p_{1}}{d t} & =1-\operatorname{sgn}\left(p_{1}\right) \sqrt{\left|p_{1}\right|}-\sqrt{\frac{\tau_{1} V_{2}}{\tau_{2} V_{1}}} \operatorname{sgn}(\Delta p) \sqrt{|\Delta p|} \\
\frac{d \Delta p}{d t} & =1-\operatorname{sgn}\left(p_{1}\right) \sqrt{\left|p_{1}\right|}-\frac{V_{1}+V_{2}}{V_{2}} \sqrt{\frac{\tau_{1} V_{2}}{\tau_{2} V_{1}}} \operatorname{sgn}(\Delta p) \sqrt{|\Delta p|}
\end{aligned}
$$

These equations can be rewritten as follows; let

$$
\begin{aligned}
p_{1} & =x \\
\Delta p & =y \\
a & =\sqrt{\frac{\tau_{1} V_{2}}{\tau_{2} V_{1}}} \\
b & =\frac{V_{1}+V_{2}}{V_{2}}
\end{aligned}
$$

Then the equations above become

$$
\begin{aligned}
& \frac{d x}{d t}=1-\operatorname{sgn}(x) \sqrt{|x|}-a \operatorname{sgn}(y) \sqrt{|y|} \\
& \frac{d y}{d t}=1-\operatorname{sgn}(x) \sqrt{|x|}-a b \operatorname{sgn}(y) \sqrt{|y|}
\end{aligned}
$$

These equations are autonomous, see [12] and [13] for example, and therefore can be reduced to a single first-order nonlinear ODE by eliminating time. Dividing these two equations, we get

$$
\frac{d y}{d x}=\frac{1-\operatorname{sgn}(x) \sqrt{|x|}-a b \operatorname{sgn}(y) \sqrt{|y|}}{1-\operatorname{sgn}(x) \sqrt{|x|}-a \operatorname{sgn}(y) \sqrt{|y|}}
$$

or

$$
\frac{d y}{d x}=1-\frac{a(b-1) \operatorname{sgn}(y) \sqrt{|y|}}{1-\operatorname{sgn}(x) \sqrt{|x|}-a \operatorname{sgn}(y) \sqrt{|y|}}
$$

Although a phase plane analysis formally starts from either of these equations, when we numerically integrate in the phase plane, we use the parametric form of the equations, Eqs. (12), and then plot the results in the phase plane.

If the two room volumes are identical and the conditions in the lower layer are the same for the example illustrated in Figure 3 then the parameters $a$ and $b$ are given 


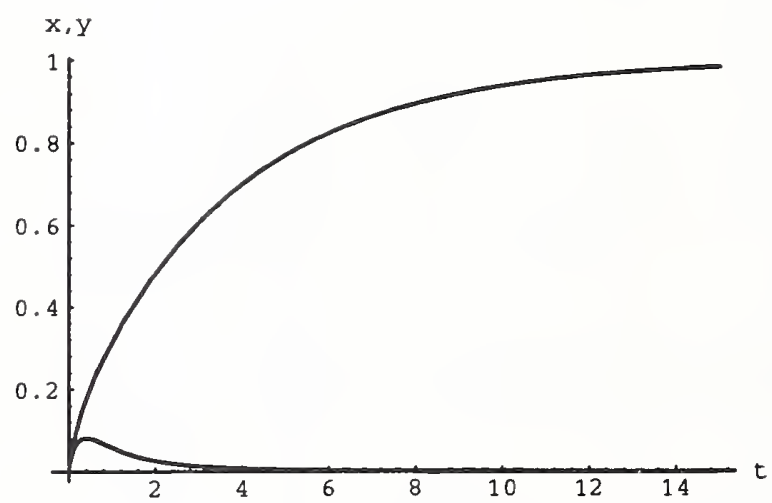

Figure 4: Solution Plot of Equation 12 for $a=1, b=2$ and Initial Conditions $x(0)=y(0)=0$

by $a=A_{\text {vent } 2} / A_{\text {vent } 1}$ and $b=2$. Large $a$ then implies that the vent connecting the two rooms is large compared to the vent connecting the first room to the outside.

In general, the fixed point of the system for $(x(t), y(t))$ is determined from the equations

$$
\begin{array}{r}
1-\operatorname{sgn}(x) \sqrt{|x|}-a \operatorname{sgn}(y) \sqrt{|y|}=0 \\
1-\operatorname{sgn}(x) \sqrt{|x|}-a b \operatorname{sgn}(y) \sqrt{|y|}=0
\end{array}
$$

and is given by $x_{0}=1, y_{0}=0$. We note an important difference, between the equations for $(x(t), y(t))$ and the usual ones encountered in phase-plane analysis [12] and [13]; these equations are not analytic around the fixed point. The fixed point is a stable one as determined by the numerical integrations described below.

Equations (12) have been integrated using the software package Mathematica. Once again, as in the one-room example, we have used the command NDSolve for this first-order nonlinear ODE system. Figure 4 shows $x(t)$ and $y(t)$ for $a=1, b=2$ with initial conditions (I.C.) $x(0)=y(0)=0$. The dimensionless pressure in room $1, x(t)$, starts at zero and increases monotonically to unity over of order ten dimensionless time units. The pressure difference between room 1 and room $2, y(t)$, starts at zero, increases to a maximum of about 0.1 at about one dimensionless time unit and then decreases to zero again. The solutions displayed are well behaved, and the numerical calculation of them encounters no particular difficulty. However, when left 


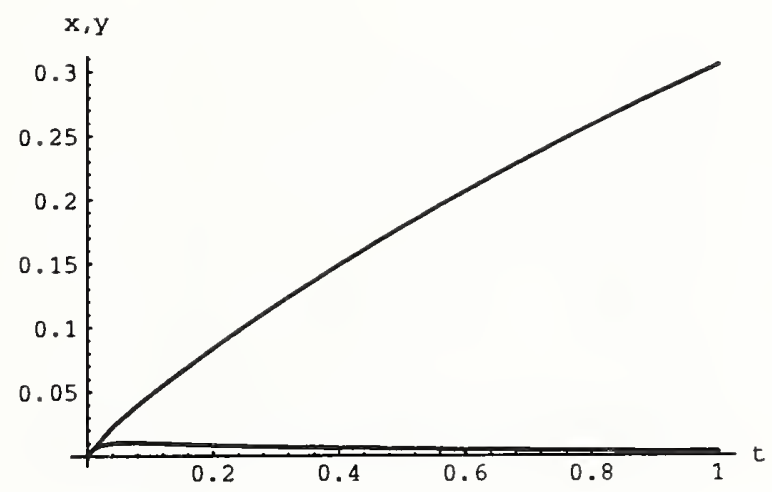

Figure 5: Solution Plot of Equation 12 for $a=4, b=2$ and Initial Conditions $x(0)=y(0)=0$

in dimensional form and coupled with the equations describing the other properties in the zone model, the equations are stiff. Therefore, in general, computation of solutions to these equations could be difficult because of the short pressure-equilibration time scale compared to the time scale for change of the other properties, such as temperature, layer height, etc.

Figure 5 shows $x(t)$ and $y(t)$ for $a=4, b=2$ with initial conditions (I.C.) $x(0)=y(0)=0$. The primary difference between these plots and those of Fig. 4 is that the solution for $y$ rises more rapidly as a function of $t$ from its initial condition to a smaller maximum of about 0.01 and then decays to zero. Large $a$ is a condition on the ratio of time scales and volumes of the two rooms. As we shall see in the following subsection, large $a$ implies that the equations are stiff and a singular perturbation analysis of the problem is applicable. We emphasize that this problem, with a relatively large value of $a$, would most probably cause difficulty using a numerical solver that did not account for stiffness of the equations. As noted above, when the pressure equations are coupled with the equations for the other properties in a zone model, they become stiff due to the disparity in time scales. Therefore, integration of these equations would most likely cause even more difficulty than integration of the previous case illustrated in Fig. 4.

Figure 6 shows $x(t)$ and $y(t)$ for $a=0.1, b=2$ with initial conditions (I.C.) $x(0)=y(0)=0$. The curves show a much more gentle time dependence than that 


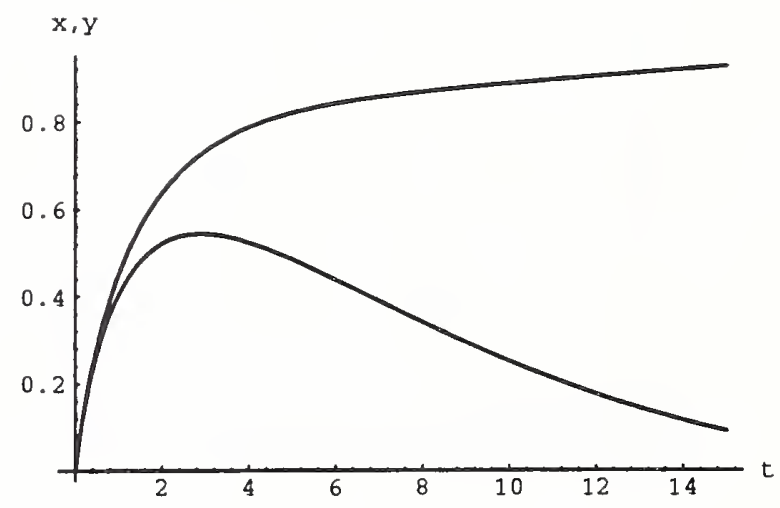

Figure 6: Solution Plot of Equation 12 for $a=0.1, b=2$ and Initial Conditions $x(0)=y(0)=0$

displayed in Fig. 5.

Figure 7 shows a phase plane plot of the solution to Eqs. (12) with $a=1, b=2$. This phase plane plot demonstrates that $x=1, y=0$ is a stable fixed point of the solution since all solutions progress toward this point as time increases. The plot was prepared by integrating Eqs. (12) for thirteen different initial conditions and then plotting each curve parametrically. All solutions reach the stable fixed point.

Figure 8 shows a phase plane plot of the solution to Eqs. (12) with $a=4, b=2$. This figure shows that the trajectories of the solution have become much more angular with nearly 45 -degree lines joined to sections of the $x$-axis. This very abrupt behavior is an indication that the Eqs. (12) are becoming stiff for the parameters chosen.

Figure 9 shows a phase plane plot of the solution to Eqs. (12) with $a=0.1, b=2$. This figure shows that the trajectories of the solution have become much smoother than those shown in either Figs. 7 or 8.

\subsection{Zero-Order Singular-Perturbation Analysis}

Return to Eqs. (11) and consider the cases when the ratio of time scales $\tau_{1} / \tau_{2}$ becomes either large or small; these cases are actually the cases of interest because physical parameters dictate that the time scale ratio will often be large or small, and these large or small numbers cause stiffness in the equations. We will not perform a formal singular perturbation analysis, but only show how the zero-order behavior of the 


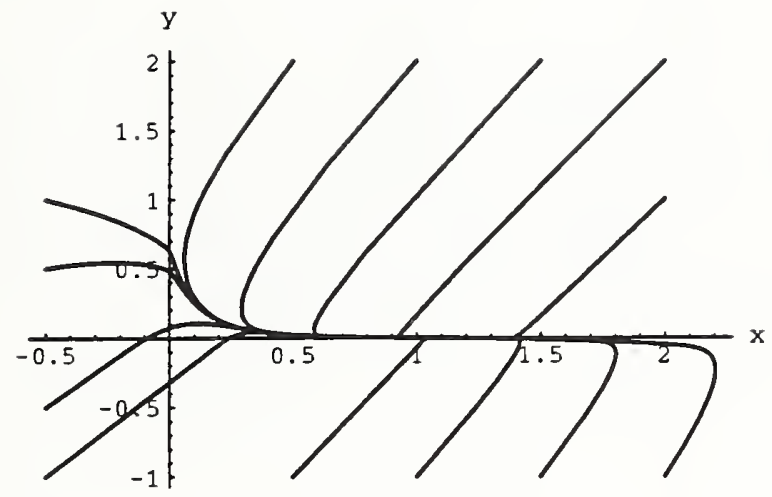

Figure 7: Phase Plane Plot of Equation 12 for $a=1, b=2$

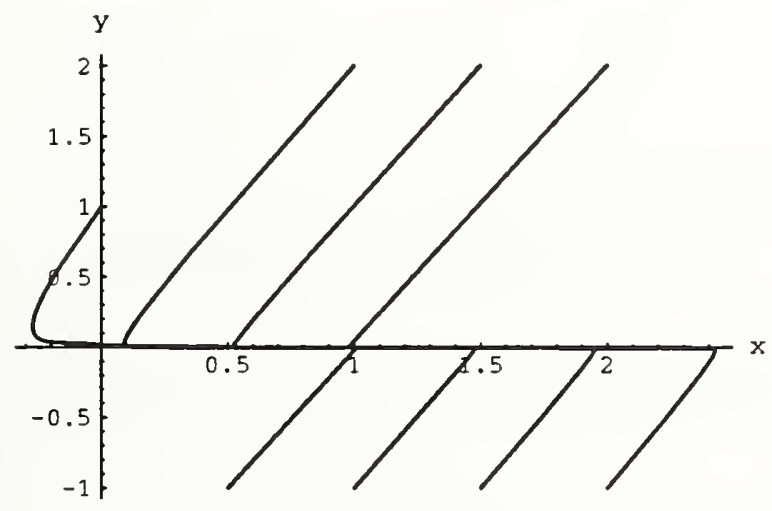

Figure 8: Phase Plane Plot of Equation 12 for $a=4, b=2$ 


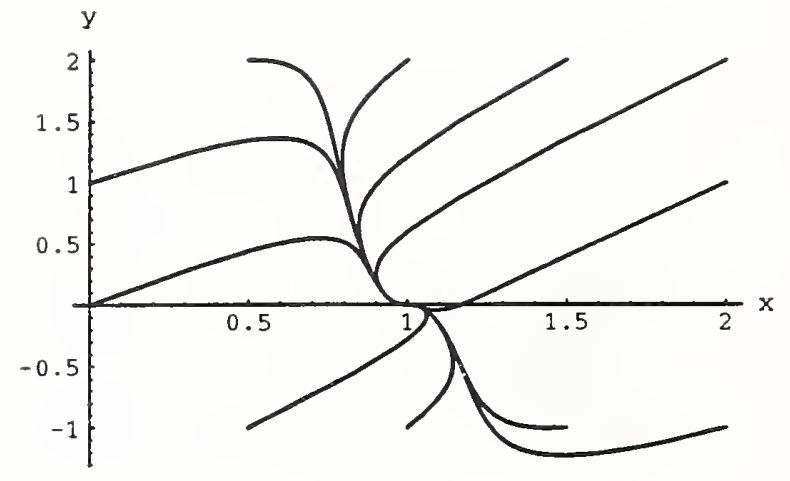

Figure 9: Phase Plane Plot of Equation 12 for $a=0.1, b=2$

system can be determined when the ratio of time scales is large or small.

First, consider the case when $\tau_{1} / \tau_{2} \gg 1$. This will occur, for example, when the area of vent two, that connecting the two rooms, is moderately large, while vent one, the vent to the outside, is a small leak. When this is the case, then, since $\tau_{1} / \tau_{2}$ multiplies the difference in room pressures, $p_{1}-p_{2} \rightarrow 0$. If we eliminate $\left(\tau_{1} / \tau_{2}\right)\left(p_{1}-p_{2}\right)$ between the two equations, we get

$$
\frac{d p_{1}}{d t}=1-\operatorname{sgn}\left(p_{1}\right) \sqrt{\left|p_{1}\right|}-\frac{V_{2}}{V_{1}} \frac{d p_{2}}{d t}
$$

If we now say $p_{2} \approx p_{1}$, then

$$
\frac{d p_{1}}{d t}=\frac{V_{1}}{V_{1}+V_{2}}\left[1-\operatorname{sgn}\left(p_{1}\right) \sqrt{\left|p_{1}\right|}\right]
$$

If we choose the proper pressure and time scales, this becomes the same as Eq. (9) for the single room with a fire and a leak, but now with a volume $V_{1}+V_{2}$, the volume of the two rooms.

Similarly, when $\tau_{1} / \tau_{2} \ll 1$, we have the case where the vent area between the two rooms is small relative to the vent area to the outside for example. In this case, we concentrate on the first of Eqs. (11) and note that the term proportional to $\tau_{1} / \tau_{2}$, the term representing the effect of the second vent, is negligible. Then, this equation becomes

$$
\frac{d p_{1}}{d t}=1-\operatorname{sgn}\left(p_{1}\right) \sqrt{\left|p_{1}\right|}
$$


the equation for the single-room case again.

\section{Conclusions}

The simple problems examined in this paper illustrate the nature of the difficulties long encountered when numerically integrating zone fire models. The pressure equations equilibrate very rapidly compared to the equations governing the other dependent variables in the zone fire models. When equations of this nature are encountered, they are referred to as stiff. The simple problems analyzed here illustrate the nature of the stiffness and demonstrate that proper nondimensionalization together with singular perturbation analysis can provide insight into the behavior of the system for parameters of interest.

The methods can be used to examine much more general problems. For example, two rooms connected with each other and with the outside in different fashion can be analyzed similarly to the two-room example presented here. In addition, some multiroom enclosures have also been analyzed using the nondimensionalization and singular perturbation methods described herein. In the limit of various leak sizes between rooms (or time scales determined by the heat source, room volume and leak rate), the equations can be shown to reduce to the one-room equation with redefined leak rates and room volumes, as was done in the two-room case illustrated above. The methods should provide an opportunity to analyze difficulties with stiffness which are encountered in more general zone fire models.

\section{References}

[1] Mitler, Henri E., "Mathematical Modeling of Enclosure Fires," Chapter 23, pp. 711-753, Numerical Approaches to Combustion Modeling, Elaine S. Oran and Jay P. Boris, eds., Vol. 135, Progress in Aeronautics and Astronautics; also, National Institute of Standards and Technology Report NISTIR 90-4294, May 1991.

[2] Morton, B.R., Taylor, G.I., and Turner, J.S., "Turbulent Gravitational Convection from Maintained and Instantaneous Sources," Proc. Roy. Soc. London A, 234 pp. 1-23, 1956.

[3] Prahl, J. and Emmons, H.W., "Fire Induced Flow Through an Opening," Combustion and Flame, 25 pp. 369-385, 1975.

[4] Rockett, J.A., "Fire Induced Gas Flow in an Enclosure," Combustion Science and Technology, 12 pp. 165-175, 1976. 
[5] Zukoski, E.E., "Development of a Stratified Ceiling Layer in the Early Stages of a Closed-Room Fire," Fire And Materials, 2 No. 2, pp. 54-62, 1978.

[6] Zukoski, E.E. and Kubota, T., "Two-layer Modeling of Smoke Movement in Building Fires," Fire And Materials, 4 No. 1, pp. 17-27, 1980.

[7] Emmons, H.W., "The Flow of Gases Through Vents," Home Fire Project Technical Report No. 75, Division of Applied Sciences, Harvard University, March $16,1987$.

[8] Quintiere, James G., "Fundamentals of Enclosure Fire 'Zone' Models," J. of Fire Prot. Engr., 1(3), pp. 99-119, 1989.

[9] Forney, Glenn P. and Moss, William F., "Analyzing and Exploiting Numerical Characteristics of Zone Fire Models," National Institute of Standards and Technology Report 4763, March 1992.

[10] Rehm, R.G. and H.R. Baum, "The Equations of Motion for Thermally Driven, Buoyant Flows", Journal of Research of the NBS, 83, pp. 297-308, May-June 1978.

[11] Wolfram, Stephen, Mathematica, A System for Doing Mathematics by Computer, Addison-Wesley, New York, 1991.

[12] Percival, I. and Richards, D., Introduction to Dynamics, Cambridge University Press, New York, 1982.

[13] Jordan, D.W. and Smith P., Nonlinear Ordinary Differential Equations, Oxford Applied Mathematics and Computing Science Series, Clarendon Press, Oxford, 1979.

[14] Cooper, L. Y., "A Mathematical Model for Estimating Available Safe Egress Time in Fires", Fire and Materials, 6, pp. 135-144, 1982.

[15] Fatunla, S. O., Numerical Methods for Initial Value Problems in Ordinary Differential Equations, Academic Press, New York, 1988. 


\section{A Zone-Fire Model Stiffness}

Stiffness is a term used to categorize a class of mathematical problems arising from physical systems that possess multiple time scales. Paradoxically, solutions to stiff problems often appear to change slowly yet have enormous computational requirements when solved using standard ie non-stiff solvers. These solvers are inefficient because they do not adequately exploit the key property of stiff systems. In stiff systems, the short time-scale phenomena approach a quasi-steady state rapidly while the other phenomena evolve on a much longer time scale.

The derivation of the differential equations for a simple zone fire model are presented here to illustrates the presense of these multiple time scales. Once the stiffness is understood, analytical techniques can be used to simplify the physical model into a non-stiff form or alternatively, special numerical procedures can be designed that exploit the properties of stiff models.

\section{A.1 A Derivation Illustrating the Presense of Stiffness}

We start with the equations of conservation of energy and mass for each of the two layers. These equations are coupled with the state equations and the various definitions. Here, tildes are used to denote dimensional quantities. The notation is that used the main body of the paper.

The energy equations are

$$
\begin{aligned}
& \frac{d \tilde{E}_{U}}{d \tilde{t}}+\tilde{p} \frac{d \tilde{V}_{U}}{d \tilde{t}}=\dot{q}_{\text {fire }}+\dot{q}_{\text {plume }}-\dot{q}_{\text {walls }} \\
& \frac{d \tilde{E}_{L}}{d \tilde{t}}+\tilde{p} \frac{d \tilde{V}_{L}}{d \tilde{t}}=-\dot{q}_{\text {vent }}-\dot{q}_{\text {plume }}
\end{aligned}
$$

The mass equations are

$$
\begin{aligned}
\frac{d \tilde{m}_{U}}{d \tilde{t}} & =\dot{m}_{\text {fire }}+\dot{m}_{\text {plume }} \\
\frac{d \tilde{m}_{L}}{d \tilde{t}} & =-\dot{m}_{\text {vent }}-\dot{m}_{\text {plume }}
\end{aligned}
$$

The state equations are for an ideal gas with constant specific-heat coefficients

$$
\begin{aligned}
\tilde{p}(\tilde{t}) & =R \tilde{\rho}_{U}(\tilde{t}) \tilde{T}_{U}(\tilde{t}) \\
\tilde{p}(\tilde{t}) & =R \tilde{\rho}_{L}(\tilde{t}) \tilde{T}_{L}(\tilde{t}) \\
\tilde{E}_{U}(\tilde{t}) & =C_{V} \tilde{m}_{U}(\tilde{t}) \tilde{T}_{U}(\tilde{t})=\frac{1}{\gamma-1} \tilde{V}_{U}(\tilde{t}) \tilde{p}(\tilde{t}) \\
\tilde{E}_{L}(\tilde{t}) & =C_{V} \tilde{m}_{L}(\tilde{t}) \tilde{T}_{L}(\tilde{t})=\frac{1}{\gamma-1} \tilde{V}_{L}(\tilde{t}) \tilde{p}(\tilde{t})
\end{aligned}
$$


The definitions are

$$
\begin{aligned}
\tilde{\rho}(\tilde{t}) & =\frac{\tilde{m}_{U}(\tilde{t})}{\tilde{V}_{U}(\tilde{t})} \\
\tilde{\rho}(\tilde{t}) & =\frac{\tilde{m}_{L}(\tilde{t})}{\tilde{V}_{L}(\tilde{t})} \\
\tilde{V}_{0} & =\tilde{V}_{L}+\tilde{V}_{U}=\text { constant } \\
\dot{q}_{\text {vent }} & =C_{p} \tilde{T}_{L}(\tilde{t}) \dot{m}_{\text {vent }} \\
\dot{m}_{\text {vent }} & =C_{\text {vent }} A_{\text {vent }} \sqrt{2 \tilde{\rho}_{L}\left(\tilde{p}-\tilde{p}_{0}\right)} \\
\dot{q}_{\text {plume }} & =C_{p} \tilde{T}_{L}(\tilde{t}) \dot{m}_{\text {plume }} \\
\dot{m}_{\text {plume }} & =\rho_{0} \sqrt{g H} H^{2}\left(Q^{*}\right)^{1 / 3} \tilde{\alpha}(\tilde{z} / H) \\
\dot{q}_{\text {walls }} & =[S+2(L+W)(H-\tilde{z})] K\left(\tilde{T}_{U}-\tilde{T}_{0}\right)
\end{aligned}
$$

$\dot{q}_{\text {fire }}$ is assumed to be a constant fire (heat) source, $\dot{q}_{\text {vent }}$ is the enthalpy loss through the vent, $\dot{q}_{\text {plume }}$ is the enthalpy pumped from the lower layer into the upper layer by the plume, and $\dot{q}_{\text {walls }}$ is the heat transfer rate to the walls. $\dot{m}_{\text {fire }}$ is the mass added by the fire, $\dot{m}_{\text {vent }}$ is the mass loss through the vent and $\dot{m}_{\text {plume }}$ is the mass pumped by the fire plume from the lower layer into the upper layer. $Q^{*}$ is the fire input parameter defined by Zukoski [5]

$$
Q^{*}=\frac{\dot{q}_{\mathrm{fire}}}{\rho_{0} C_{p} T_{0} \sqrt{g H} H^{2}}
$$

and $\rho_{0}, T_{0}$ are reference density and temperature, $g$ is the acceleration of gravity and $H$ is the height of the enclosure. $S=L W$ is the floor area of the enclosure, where $L$ is the length of the enclosure and $W$ is its width. $K$ is a heat transfer coefficient for heat transfer from the gas to the enclosure walls. Then $\tilde{V}_{0}=L W H$. The control volumes are around the upper and the lower layers, and the sum of the two control volumes is the total volume of the enclosure, which is constant.

An equation for the pressure is found by adding the energy equations for the two layers, taking account of the equation of state for the ideal gas.

$$
\begin{gathered}
\frac{d \tilde{E}_{U}}{d t}+\frac{d \tilde{E}_{L}}{d t}+\tilde{p} \frac{d}{d \tilde{t}}\left(\tilde{V}_{U}+\tilde{V}_{L}\right)=\dot{q}_{\text {fire }}-\dot{q}_{\text {vent }}-\dot{q}_{\text {walls }} \\
\frac{d}{d \tilde{t}}\left[\tilde{p}\left(\tilde{V}_{U}+\tilde{V}_{L}\right)\right]=(\gamma-1)\left(\dot{q}_{\text {fire }}-\dot{q}_{\text {vent }}-\dot{q}_{\text {walls }}\right) \\
\frac{d \tilde{p}}{d \tilde{t}}=\frac{\gamma-1}{V_{0}}\left(\dot{q}_{\text {fire }}-\dot{q}_{\text {vent }}-\dot{q}_{\text {walls }}\right)
\end{gathered}
$$


Here the notation is as defined earlier except that $\tilde{p}$ is the pressure in the room relative to the ambient pressure outside the room $\tilde{p}=\tilde{p}_{\text {enc }}-\tilde{p}_{\text {atm }}$, and $\tilde{t}$ is the time.

The upper-layer energy equation and the upper-layer mass equation combine to give an equation for the upper-layer temperature. The upper-layer energy equation becomes:

$$
\begin{aligned}
\frac{1}{\gamma-1} \frac{d}{d \tilde{t}}\left(\tilde{p} \tilde{V}_{U}\right)+\tilde{p} \frac{d \tilde{V}_{U}}{d \tilde{t}} & =\dot{q}_{\text {fire }}+\dot{q}_{\text {plume }}-\dot{q}_{\text {walls }} \\
\frac{1}{\gamma-1}\left(\tilde{V}_{U} \frac{d \tilde{p}}{d \tilde{t}}+\tilde{p} \frac{d \tilde{V}_{U}}{d \tilde{t}}\right)+\tilde{p} \frac{d \tilde{V}_{U}}{d \tilde{t}} & =\dot{q}_{\text {fire }}+\dot{q}_{\text {plume }}-\dot{q}_{\text {walls }} \\
\frac{\gamma}{\gamma-1} \frac{d}{d \tilde{t}}\left(\tilde{p} \tilde{V}_{U}\right)-\tilde{V}_{U} \frac{d \tilde{p}}{d \tilde{t}} & =\dot{q}_{\text {fire }}+\dot{q}_{\text {plume }}-\dot{q}_{\text {walls }}
\end{aligned}
$$

The upper-layer mass equation is

$$
\begin{aligned}
\frac{d \tilde{m}_{U}}{d t}=\frac{d \tilde{\rho}_{U} \tilde{V}_{U}}{d \tilde{t}} & =\dot{m}_{\text {plume }}+\dot{m}_{\text {fire }} \\
\frac{\gamma}{\gamma-1} R \tilde{T}_{U} \frac{d}{d \tilde{t}}\left(\tilde{\rho}_{U} \tilde{V}_{U}\right) & =C_{p} \tilde{T}_{U}\left(\dot{m}_{\text {plume }}+\dot{m}_{\text {fire }}\right) \\
\frac{\gamma}{\gamma-1}\left[\frac{d}{d \tilde{t}}\left(R \tilde{T}_{U} \tilde{\rho}_{U} \tilde{V}_{U}\right)-\tilde{\rho}_{U} \tilde{V}_{U} \frac{d}{d \tilde{t}}\left(R \tilde{T}_{U}\right)\right] & =C_{p} \tilde{T}_{U}\left(\dot{m}_{\text {plume }}+\dot{m}_{\text {fire }}\right) \\
\frac{\gamma}{\gamma-1}\left[\frac{d}{d \tilde{t}}\left(\tilde{p} \tilde{V}_{U}\right)-\tilde{\rho}_{U} \tilde{V}_{U} R \frac{d}{d \tilde{t}}\left(\tilde{T}_{U}\right)\right] & =C_{p} \tilde{T}_{U}\left(\dot{m}_{\text {plume }}+\dot{m}_{\text {fire }}\right)
\end{aligned}
$$

Combining the upper-layer energy and mass equations

$$
\frac{\gamma}{\gamma-1} R \tilde{\rho}_{U} \tilde{V}_{U} \frac{d}{d \tilde{t}}\left(\tilde{T}_{U}\right)=\tilde{V}_{U} \frac{d \tilde{p}}{d \tilde{t}}+\dot{q}_{\text {fire }}+\dot{q}_{\text {plume }}-\dot{q}_{\text {walls }}-C_{p} \tilde{T}_{U}\left(\dot{m}_{\text {plume }}+\dot{m}_{\text {fire }}\right)
$$

Now noting Eq.(28) and that $C_{p}=\frac{\gamma}{\gamma-1} R$,

$$
C_{p} \tilde{\rho}_{U} \tilde{V}_{U} \frac{d}{d \tilde{t}}\left(\tilde{T}_{U}\right)=\tilde{V}_{U} \frac{d \tilde{p}}{d \tilde{t}}+\dot{q}_{\text {fire }}-\dot{q}_{\text {walls }}-C_{p}\left(\tilde{T}_{U}-\tilde{T}_{L}\right) \dot{m}_{\text {plume }}-C_{p} \tilde{T}_{U} \dot{m}_{\text {fire }}
$$

Finally, the lower-layer mass equation is

$$
\frac{d \tilde{m}_{L}}{d t}=\frac{d \tilde{\rho}_{L} \tilde{V}_{L}}{d \tilde{t}}=-\dot{m}_{\text {plume }}-\dot{m}_{\text {vent }}
$$

Rewrite the three equations for the pressure, the upper layer gas temperature and the lower layer mass.

$$
\frac{d \tilde{p}}{d \tilde{t}}=\frac{\gamma-1}{V_{0}}\left(\dot{q}_{\text {fire }}-\dot{q}_{\text {vent }}-\dot{q}_{\text {walls }}\right)
$$




$$
\begin{aligned}
C_{p} \tilde{\rho}_{U} \tilde{V}_{U} \frac{d}{d \tilde{t}}\left(\tilde{T}_{U}\right) & =\tilde{V}_{U} \frac{d \tilde{p}}{d \tilde{t}}+\dot{q}_{\text {fire }}-\dot{q}_{\text {walls }}-C_{p}\left(\tilde{T}_{U}-\tilde{T}_{L}\right) \dot{m}_{\text {plume }}-C_{p} \tilde{T}_{U} \dot{m}_{\text {fire }} \\
\frac{d \tilde{\rho}_{L} \tilde{V}_{L}}{d \tilde{t}} & =-\dot{m}_{\text {plume }}-\dot{m}_{\text {vent }}
\end{aligned}
$$

Use much of the notation and the scales of Zukoski to define dimensionless quantities. If $\tilde{z}$ is the dimensional layer height, then

$$
\begin{aligned}
z & =\tilde{z} / H \\
V_{L} & \equiv \tilde{V}_{L} / \tilde{V}_{0}=z \\
V_{U} & \equiv \tilde{V}_{U} / \tilde{V}_{0}=1-z
\end{aligned}
$$

Define the reference pressure $p_{0}$, temperature $T_{0}$ and density $\rho_{0}$ to be related by $p_{0}=R \rho_{0} T_{0}$.

$$
\begin{aligned}
\rho_{L} & \equiv \tilde{\rho}_{L} / \tilde{\rho}_{0} \\
\rho_{U} & \equiv \tilde{\rho}_{U} / \tilde{\rho}_{0} \\
T_{L} & \equiv \tilde{T}_{L} / \tilde{T}_{0} \\
T_{U} & \equiv \tilde{T}_{U} / \tilde{T}_{0}
\end{aligned}
$$

The time scale defined by Zukoski for nondimensionalization is

$$
t_{Z} \equiv \sqrt{H / g}\left(S / H^{2}\right)
$$

The only tricky feature of the current nondimensionalization is that the pressure must be defined carefully. Let $\tilde{p} \equiv \tilde{p}_{0}+\Delta \tilde{p}$; i.e., the pressure is the reference pressure plus the overpressure in the enclosure. The overpressure must be made dimensionless with respect to $\tilde{p}_{\infty}$ where

$$
\tilde{p}_{\infty}=\left(\frac{\dot{q}_{\text {fire }}}{C_{p} T_{L} c_{\text {vent }} A_{\text {vent }} \sqrt{2 \rho_{L}}}\right)^{2}
$$

Then

$$
p \equiv \tilde{p} / \tilde{p}_{0}=1+\Delta \tilde{p} / \tilde{p}_{0}=1+\left(\tilde{p}_{\infty} / \tilde{p}_{0}\right) \Delta p=1+\epsilon \Delta p
$$

where $\epsilon \equiv\left(\tilde{p}_{\infty} / \tilde{p}_{0}\right)$.

Rewrite the mass and heat sources and sinks as follows:

$$
\dot{m}_{\text {fire }}=\tilde{\rho}_{0} \sqrt{g H} H^{2} M
$$




$$
\begin{aligned}
\dot{q}_{\text {fire }} & =\tilde{\rho}_{0} C_{p} \tilde{T}_{0} \sqrt{g H} H^{2} Q^{*} \\
\dot{m}_{\text {vent }} & =\tilde{\rho}_{0} \sqrt{g H} H^{2} Q_{V} \sqrt{\epsilon} \sqrt{\rho_{L} \Delta p} \\
\dot{q}_{\text {vent }} & =C_{p} \tilde{T}_{0} T_{L} \dot{m}_{\text {vent }} \\
& =C_{p} \tilde{T}_{0} \tilde{\rho}_{0} \sqrt{g H} H^{2} Q_{V} \sqrt{\epsilon} T_{L} \sqrt{\rho_{L} \Delta p} \\
\dot{m}_{\text {plume }} & =\tilde{\rho}_{0} \sqrt{g H} H^{2}\left(Q^{*}\right)^{1 / 3} \tilde{\alpha} z^{5 / 3} \\
\dot{q}_{\text {plume }} & =C_{p} \tilde{T}_{0} \tilde{\rho}_{0} \sqrt{g H} H^{2} T_{L}\left(Q^{*}\right)^{1 / 3} \tilde{\alpha} z^{5 / 3} \\
\dot{q}_{\text {walls }} & =[S+2(L+W)(H-\tilde{z})] K\left(\tilde{T}_{U}-\tilde{T}_{0}\right) \\
& =C_{p} \tilde{T}_{0} \tilde{\rho}_{0} \sqrt{g H} H^{2} Q_{W}\left[1+2 \frac{(L+W) H}{S}(1-z)\right]\left(T_{U}-1\right)
\end{aligned}
$$

where

$$
\begin{aligned}
Q^{*} & =\frac{\dot{q}_{\text {fire }}}{\rho_{0} C_{p} T_{0} \sqrt{g H} H^{2}} \\
Q_{V} & =\frac{c_{\text {vent }} A_{\text {vent }} \sqrt{2 \rho_{0} p_{0}}}{\tilde{\rho}_{0} \sqrt{g H} H^{2}} \\
& =\sqrt{\frac{2}{\gamma}} c_{\text {vent }} \frac{A_{\text {vent }}}{H^{2}} \frac{c_{\text {sound }}}{\sqrt{g H}} \\
Q_{W} & =\frac{S K}{C_{p} \tilde{\rho}_{0} \sqrt{g H} H^{2}}
\end{aligned}
$$

The equations in dimensionless form then become:

$$
\begin{aligned}
\epsilon \frac{d \Delta p}{d t}= & \gamma\left[Q^{*}-Q_{V} \sqrt{\epsilon} T_{L} \sqrt{\rho_{L} \Delta p}-Q_{W}\left[1+2 \frac{(L+W) H}{S}(1-z)\right]\left(T_{U}-1\right)\right] \\
\rho_{U} V_{U} \frac{d T_{U}}{d t}= & \epsilon \frac{\gamma-1}{\gamma} V_{U} \frac{d \Delta p}{d t}+Q^{*}-Q_{W}\left[1+2 \frac{(L+W) H}{S}(1-z)\right]\left(T_{U}-1\right) \\
& -\left(Q^{*}\right)^{1 / 3} \tilde{\alpha} Z^{5 / 3}\left(T_{U}-T_{L}\right)-M T_{U} \\
\frac{d \rho_{L} V_{L}}{d t}= & -\left(Q^{*}\right)^{1 / 3} \tilde{\alpha} Z^{5 / 3}-Q_{V} \sqrt{\epsilon} \sqrt{\rho_{L} \Delta p}
\end{aligned}
$$

The purpose of the derivation presented here is to demonstrate that the equations describing a very simple case of a zone-fire model are stiff. Zukoski [5] has presented numerical estimates of the magnitudes of the various parameters which appear in these equations. In particular, he has demonstrated that leaks generally are large enough in most enclosure fire scenarios that the overpressure which can develop is rather small. (In fact, Zukoski uses this fact to ignore any overpressure and make a quasisteady approximation for the pressure, assuming that it equilibrates instantaneously 
to the pressure outside the enclosure, during the enclosure-filling process. If the overpressure were to become significant, in most cases there would be a structural failure such as a window breaking to relieve this overpressure. A possible exception might be any enclosure which was designed to accommodate large overpressures, such as a compartment in a submarine for example.) When the leak is large enough to sustain only small values of overpressure, then $\epsilon \equiv \frac{\bar{p}_{\infty}}{\tilde{p}_{0}} \ll 1$ is a small parameter. Using equation (32) with $\dot{q}_{\text {fire }}=100,000 \mathrm{~W}, A_{\text {vent }}=1 \mathrm{~m}^{2}, c_{\text {vent }}=.68$ and ambient density and temperature, $\epsilon \approx 10^{-6}$. Since this small parameter multiplies the time derivative of the overpressure, the system of equations is stiff, and the culprit is the overpressure equation.

Other observations can be made from this dimensionless system of equations. However, we will note only one. The state equation is $p=1+\frac{\bar{p}_{\infty}}{\tilde{p}_{0}} \Delta p=\rho_{L} T_{L}=\rho_{U} T_{U}$. When $\epsilon=\frac{\tilde{p}_{\infty}}{\tilde{p}_{0}} \ll 1$, then $p \approx 1$ and $\rho_{L} T_{L}=1, \rho_{U} T_{U}=1$. Then $\rho_{L} \approx 1$ and $T_{L} \approx 1$.

\section{A.2 Some Numerical Considerations}

As pointed out in [9] many different (but analytically equivalent) sets of differential equations can be derived to form a zone fire model. Further, the numerical difficulties encountered in these models because of stiffness can not be avoided simply by exchanging the pressure equation for some other equation such as temperature, density, or internal energy. As shown in Table 1, each zone modeling differential equation contains a $\frac{d P}{d t}$ term. For a one room zone fire model, if the pressure is computed using equation (8), the formula for asymptotic pressure rise, and $\frac{d P}{d t}$ is removed from the other modeling differential equations, then the resulting approximate differential equations are not stiff and a standard nonstiff solver may be used. This is essentially why one room models such as ASET [14] can use non-stiff solvers in their solution. For multi-room zone fire models, a non-linear set of equations need to be solved to obtain the quasi-steady state pressures for each room. However, the class of problems that can be solved is reduced since large pressure fluctuations can not be modeled properly.

The curious aspect of stiff differential equations is that the solution appears to be changing slowly and yet the computational costs of computing this solution are enormous when using nonstiff differential equation solvers such as Runge-Kutta methods. The question then is why does it cost so much to solve a problem whose solution changes slowly? To maintain stability, a nonstiff solver must use a stepsize that is small enough to track the part of the solution corresponding to the shortest time scale even when this solution component decays rapidly to some quasi-steady value. This stepsize is much smaller than required to accurately track the desired part of the solution which corresponds to one of the longer time scales. So for stiff problems the choice of stepsize is dominated by considerations of stability, not accuracy. 
There is no one definition of stiffness that is universally applied to initial value problems. One that is commonly applied is the following (see [15]). An initial value problem, $d y / d t=f(y, t), y\left(t_{0}\right)=y_{0}$ is called stiff if the eigenvalues, $\lambda_{j}=u_{j}+i v_{j}$, $j=1, \ldots, N$ of the Jacobian, $f_{y}$, satisfy

$$
u_{j}<0, j=1, \ldots, N,
$$

and

$$
\max _{1 \leq j \leq N}\left(\left|u_{j}\right|\right) \gg \min _{1 \leq j \leq N}\left(\left|u_{j}\right|\right) \text {. }
$$

In our case, the eigenvalues with large negative real parts correspond to the short time scale phenomena (room pressures).

\section{B Mathematica Commands Used to Perform Cal- culations and to Produce Figures}

This appendix documents the commands used to both perform the calculations and to produce the plots illustrated in this report. These calculations were performed using Mathematica[11]. The equivalent analysis using traditional methods involving FORTRAN would have certainly taken much longer; perhaps weeks instead of several hours. The simple models for the vent and fire used to produce phase plane plots can be made more complex and hence more realistic by adding a few "equations" to the following Mathematica code.

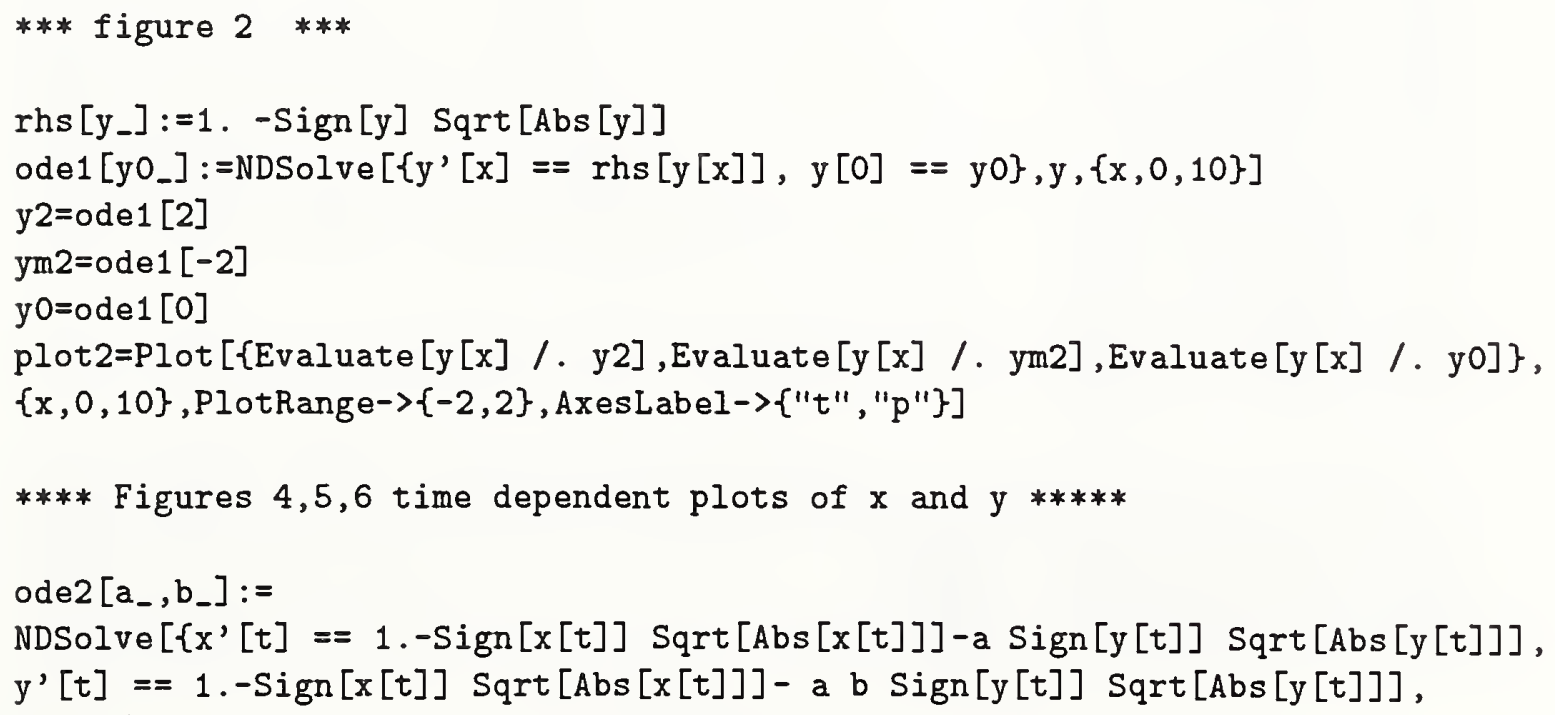




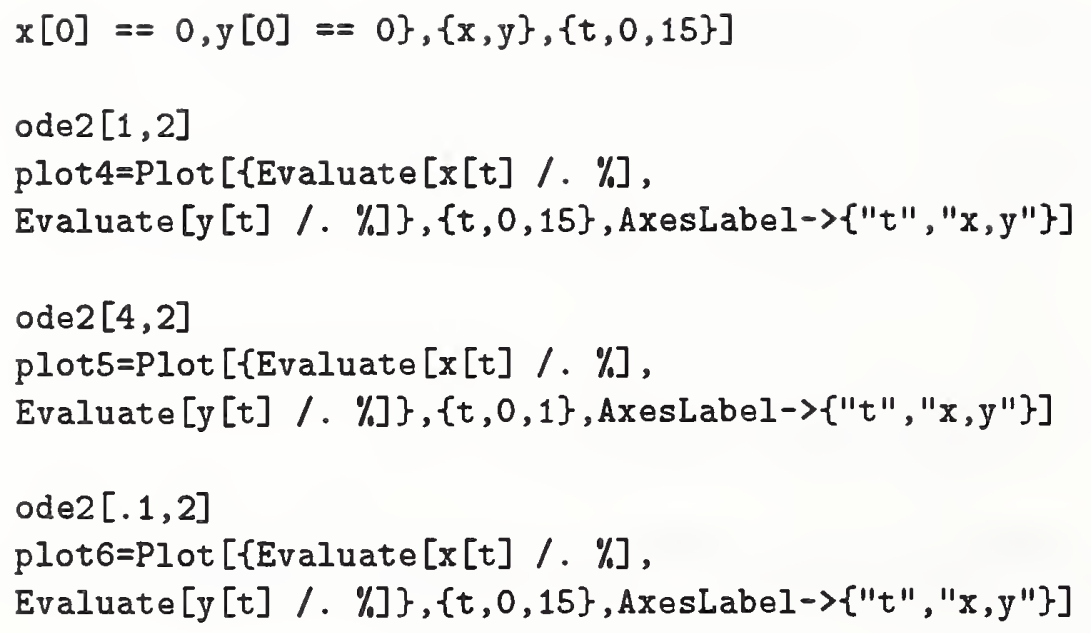


Evaluate $[\{x[t], y[t]\} / . x y p 5]$,
Evaluate $[\{x[t], y[t]\} / . x y p 6]$,
Evaluate $[\{x[t], y[t]\} / . x y p 7]$,
Evaluate $[\{x[t], y[t]\} / . x y n 1]$,
Evaluate $[\{x[t], y[t]\} / . x y n 2]$,
Evaluate $[\{x[t], y[t]\} / . x y n 3]$,
Evaluate $[\{x[t], y[t]\} / x y n 4]$,
Evaluate $[\{x[t], y[t]\} / . x y n 5]$,
Evaluate $[\{x[t], y[t]\} / . x y n 6]\}$,
$\{t, 0,15\}, P l o t R a n g e->$ All, AxesLabel->\{"x", $y "\}]$
*** Phase Space Parametric Plot - Figure $8 * * *$

xyp2 $2=\operatorname{ode} 3[4,2,1,2]$

хур $3=\operatorname{ode} 3[4,2,2,2]$

xyp $5=0 \operatorname{de} 3[4,2,1.5,2]$

xyp $6=$ ode $3[4,2,0,1]$

xyn $1=\operatorname{ode} 3[4,2,1,-1]$

xyn $3=\operatorname{ode} 3[4,2, .5,-1]$

$\operatorname{xyn} 4=\operatorname{ode} 3[4,2,1.5,-1]$

xyn5 $=\operatorname{ode} 3[4,2,2,-1]$

plot8=ParametricPlot [

$\{$ Evaluate $[\{x[t], y[t]\} /$ xyp2 $]$,

Evaluate $[\{x[t], y[t]\} / . x y p 3]$,

Evaluate $[\{x[t], y[t]\} / x y p 5]$,

Evaluate $[\{x[t], y[t]\} / . x y p 6]$,

Evaluate $[\{x[t], y[t]\} /$. xyn 1$]$,

Evaluate $[\{x[t], y[t]\} / x y n 3]$,

Evaluate $[\{x[t], y[t]\} / . x y n 4]$,

Evaluate $[\{x[t], y[t]\} / x y n 5]\}$,

$\{t, 0,15\}, P l o t R a n g e->$ All, AxesLabel->\{"x", "y" $\}]$

*** Phase Space Parametric Plot - Figure 9 ***

$\operatorname{xyp} 1=\operatorname{ode} 3[.1,2,0,0]$

xyp $2=\operatorname{ode} 3[.1,2,1,2]$

xyp $3=\operatorname{ode} 3[.1,2,2,2]$

xyp $4=0 \operatorname{de} 3[.1,2, .5,2]$

xyp5 $=$ ode $3[.1,2,1.5,2]$ 


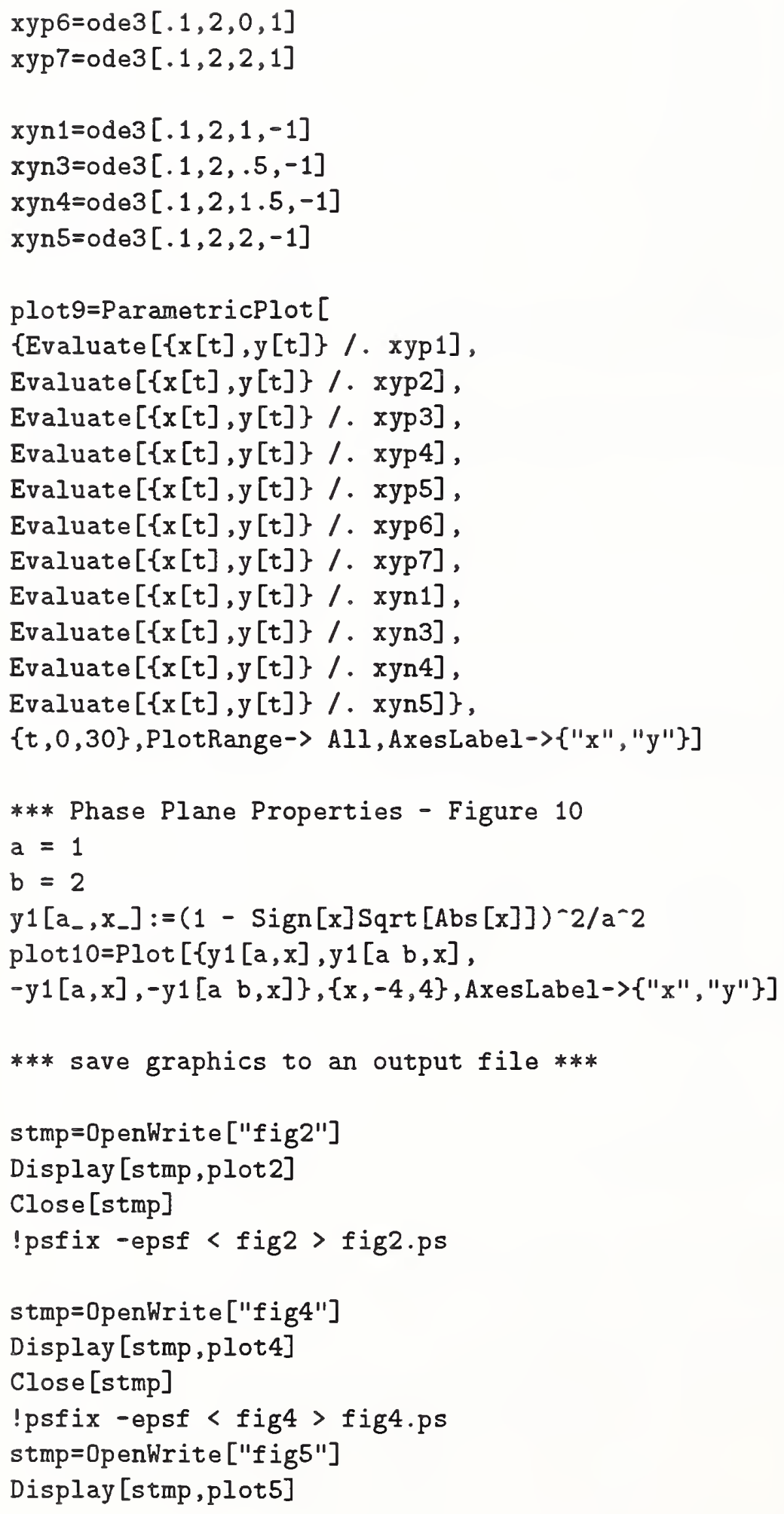




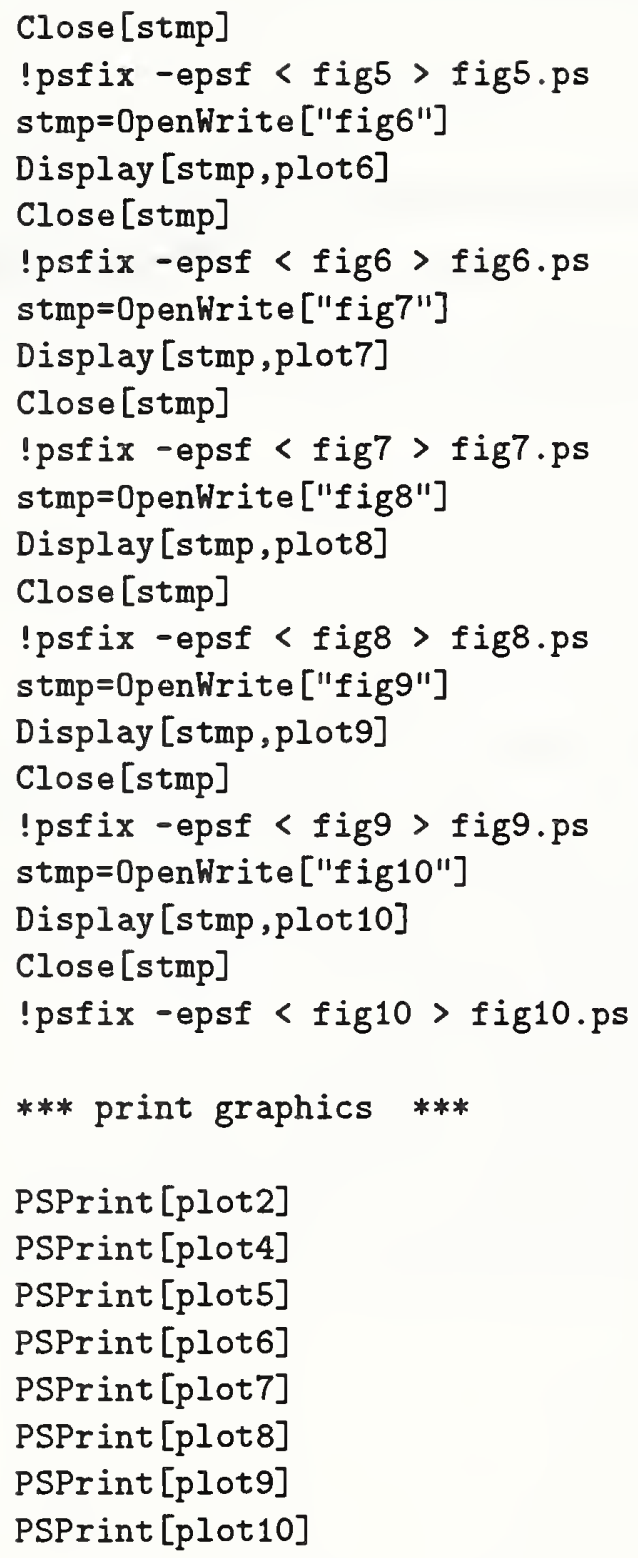





\section{BIBLIOGRAPHIC DATA SHEET}

4. TILEAND SUBTITLE

A Note on the Pressure Equations Used in Zone Fire Modeling

5. AUTHOA(S)

R.G. Rehm and G.P. Forney

6. PERFOAMINO ORCANIZATION (IF JOINT OH OTHER THAN MIST, SEE INSTRUCTIONS) U.S. DEPARTMENT OF COMUERCE MATIONAL INSTITUTE OF STANDARDS AND TECHNOLOOY CATHERSBURG, WD 20890

SPONSORIMO ORQANIZATIOK RAME AHD COMPLETE ADDRESS (STREET, CITY, STATE, ZIP)

11. ABSTRACT (A 200-WOAD OA LESS FACTUAL SUMMAAY OF MOST SIONIFICANT INFORMATION. IF DOCUMENT INCLUDES A SIONIFICANT BIBUOGAAPHY OR UTERATURE SUAVEY, MENTION TT HERE)

Examples of simple zone fire models are analyzed. These models illustrate the nature of the numerical problems commonly encountered in zone models of enclosure fires. Often these difficulties arise in the solution of the equations for the pressure in connected rooms, and they arise because the pressure equilibrates much more rapidly than other dynamical variables. Since these models are very simple, analytical techniques can be applied and some insight gained regarding the nature of these problems. The models consist of ordinary differential equations coupled with algebraic equations. Singular perturbation methods and phase plane analysis, together with numerical integration of the appropriately nondimensionalized equations, are employed to examine the stiff nature of the equations associated with these models. We conclude that many of the difficulties associated with numerical integration of zone fire models in general may be circumvented by appropriate analysis of the zone fire model equations.

12. KEY WORDS (6 TO 12 ENTRIES; ALPHABETICAL ORDEA; CAPITAUZE ONLY PAOPER NAMES; AMD SEPARATE KEY WORDS BY SEMICOLONS) fire models; mathematical models; numerical integration; ordinary differential equations; phase plane analysis; singular perturbation analysis; zone models

FOR OFFICIAL DISTRIBUTION. DO NOT RELEASE TO MATIONAL TECHNICAL INFORMATION SERVCE (NTIS). 


$$
\text { . }
$$



